\title{
Nuevas especies de la familia Marginellidae (Mollusca: Neogastropoda: Muricoidea) de Venezuela
}

\author{
New species of the family Marginellidae (Mollusca: Neogastropoda: Muricoidea) from Venezuela
}

\author{
Manuel Caballer ${ }^{1}$, José Espinosa ${ }^{2}$, Jesús Ortea $^{3}$ y Samuel Narciso ${ }^{4}$
}

\author{
${ }^{1}$ Centro de Oceanología y Estudios Antárticos, Instituto Venezolano de Investigaciones Científicas, Ctra. Panamericana Km \\ 11, Miranda, Venezuela. manuelcaballergutierrez@hotmail.com \\ ${ }^{2}$ Instituto de Oceanología, Avda. $1^{\mathrm{a}} \mathrm{n}^{\circ} 18406$, E. 184 y 186, Playa, La Habana, Cuba \\ ${ }^{3}$ Departamento de Biología de Organismos y Sistemas, Universidad de Oviedo, C/ Catedrático Rodrigo Uría s/n. 33071, \\ Oviedo, España \\ ${ }^{4}$ FUDENA, Fundación para la Defensa de la Naturaleza, Calle Carabobo s/n, Chichiriviche, Falcón, Venezuela
}

\begin{abstract}
To date, 10 species of the genus Volvarina and one species of the genus Hyalina have been cited in Venezuela, but only 2 of them are certain. In this paper 4 new species of mollusks of the family Marginellidae are described, based on specimens collected from 3 different locations: La Tortuga Island, Morrocoy National Park and Aves Island. Additionally, the records in Venezuela of all the species of both genus and the validity of Caribeginella flormarina, synonymized with Hyalina pallida, are discussed. Illustrations of the living animals for all the species are included, so as new photos of the shell of the Neotype of $H$. pallida, housed in the Natural History Museum in London. The new species are characterized by: Volvarina morrocoyensis Caballer, Espinosa \& Ortea new species; 5 brownish bands in the shell, paired parallel plications, white body with red spots, mantle with black spots grouped in bands. Volvarina monchoi Caballer, Espinosa \& Ortea new species; white shell with a brown band, uneven plications, white body without spots. Volvarina avesensis Caballer, Espinosa \& Ortea new species; 3 orange bands in the shell, parallel plications, orange body, lacking spots, mantle with dull spots. Hyalina nelsyae new species; 3 dull bands in the shell, posterior plications divergent, anterior plications quasi-parallel, body with reddish spots forming a diamond in the tail, mantle with reddish spots forming 3 bands.
\end{abstract}

Key words: Volvarina, Hyalina, endemism, neotype, Caribeginella flormarina, Caribbean

Resumen.- Hasta la fecha se han citado en Venezuela 10 especies del género Volvarina y una del género Hyalina, aunque de ellas solamente 2 se conocen con certeza. En este trabajo se describen 4 especies nuevas de moluscos de la familia Marginellidae a partir de ejemplares recolectados en 3 localidades diferentes: la isla de La Tortuga, el Parque Nacional Morrocoy e Isla de Aves. Además, se discute la validez de las citas de Venezuela y la de Caribeginella flormarina Espinosa \& Ortea, 1998, sinonimizada con Hyalina pallida. Se aportan ilustraciones de los animales vivos de las 4 especies y también nuevas fotos de la concha del neotipo de H. pallida, depositado en el Museo de Historia Natural de Londres. Las nuevas especies se caracterizan por: Volvarina morrocoyensis Caballer, Espinosa \& Ortea especie nueva; 5 bandas pardas en la concha, pliegues paralelos 2 a 2, cuerpo blanco con manchas rojas, manto con manchas negras formando bandas. Volvarina monchoi Caballer, Espinosa \& Ortea especie nueva; concha blanca con una banda parda, pliegues desiguales, cuerpo blanco sin manchas. Volvarina avesensis Caballer, Espinosa \& Ortea especie nueva; 3 bandas anaranjadas en la concha, pliegues paralelos, cuerpo anaranjado sin manchas, manto con manchas castañas. Hyalina nelsyae especie nueva; 3 bandas pardas en la concha, pliegues posteriores divergentes, anteriores casi paralelos, cuerpo con manchas rojizas formando un diamante en la cola, manto con manchas rojizas en 3 bandas.

Palabras clave: Volvarina, Hyalina, endemismo, neotipo, Caribeginella flormarina, Caribe

\section{INTRODUCCIÓN}

La familia Marginellidae Fleming, 1828 está compuesta por moluscos marinos de concha lisa, brillante, generalmente con 4 pliegues en la columela y desarrollo intracapsular (Gofas 2011), ya que sus especies ponen un único huevo de tamaño grande y con mucho albumen del que sale un solo ejemplar que crece hasta el tamaño adulto (Coovert \& Coovert 1995, Ponder 1998), teniendo, por lo tanto, una capacidad de dispersión limitada y tendencia al endemismo. Está representada por varios géneros en el Caribe, siendo Volvarina Hinds, 1844 e Hyalina Schumacher, 1817 dos de los más comunes en los inventarios de moluscos (Espinosa \& Ortea 2001, Espinosa et al. 2006, Yidi \& Sarmiento 2011). Las especies de Volvarina se caracterizan por presentar una concha 
sólida, pequeña a mediana, ovoide, blanca o naranja a parda con bandas de color, la espira media a elevada, la apertura estrecha, el labio engrosado y liso, la columela con 3 ó 4 pliegues, a veces hasta 6 , ocupando la mitad o menos de la longitud de la apertura, y animales con rádula (Coovert \& Coovert 1995, Caballer et al. obs. pers.). Las especies de Hyalina se caracterizan por presentar una concha fina, a veces bastante frágil, que puede ser de mediana a grande, en general alargada y de lados rectos, con un hombro moderado, blanca translúcida hasta brillantemente coloreada, la espira plana a corta, la apertura amplia, el labio fino y liso, la columela con 2 a 4 pliegues, ocupando la mitad o menos de la longitud de la apertura, y animales sin rádula (Coovert \& Coovert 1995, Caballer et al. obs. pers.).

En Venezuela, la lista de las 10 especies de Volvarina citadas por diversos autores ha sido recopilada por Caballer et al. (2009). No obstante, debido a la carencia de ilustraciones o descripciones que las avalen, solamente 2 de ellas, Volvarina ivic Caballer, Espinosa \& Ortea, 2009 y Volvarina latortuga Caballer, Espinosa \& Ortea, 2009, se conocen con certeza.

Los registros de Hyalina pallida (Linnaeus, 1758) en las localidades de Sucre, Ensenada de Turpialito, Golfo de Santa Fé, Archipiélago de Los Roques, Isla La Orchila e Isla de Aves (Gibson-Smith 1972, Macsotay 1975, Fernández \& Jiménez 2006, 2007), deben ser revisados teniendo en cuenta la escasa capacidad de dispersión de estos animales y los datos aportados por las nuevas colectas. En este sentido, los ejemplares de Isla de Aves se describen como una nueva especie del género en este trabajo.

Diversos muestreos realizados en la isla de La Tortuga, en el Parque Nacional Morrocoy y en Isla de Aves, Venezuela, con el objetivo de contribuir a esclarecer la sistemática de la familia Marginellidae en el país, han tenido como resultado la captura de ejemplares de 4 especies; 3 del género Volvarina y una del género Hyalina, cuyos caracteres no se corresponden con la diagnosis de ninguna de las conocidas en el mar Caribe y que por ello se describen como nuevas para la ciencia.

\section{Materiales y MÉtodos}

Los especímenes fueron capturados manualmente mediante buceo en isla La Tortuga, en el Parque Nacional Morrocoy (PNM) y en el Refugio de Fauna Silvestre 'Isla de Aves' (RFSIA) entre 2009 y 2012. Los ejemplares se estudiaron en vivo usando un microscopio estereoscópico Carl Zeiss ${ }^{\circledR}$, tomando datos de su anatomía externa, coloración y medidas de la longitud y la anchura de la concha. Posteriormente fueron fotografiados y conservados en etanol al $96 \%$. Una vez preservados se fotografiaban también sus conchas, estudiando las proporciones entre la protoconcha y la teleoconcha. En el texto, las medidas de los ejemplares examinados aparecen entre paréntesis; primero la longitud y después la anchura de la concha (longitud x anchura).

Los ejemplares fueron depositados en las siguientes instituciones: Fundación para la Defensa de la Naturaleza, Falcón, Venezuela (FUDENA); Sección de Organismos Marinos de las Colecciones Biológicas del Instituto Venezolano de Investigaciones Científicas, Miranda, Venezuela (SOM-IVIC; número de registro: 028). Adicionalmente, con fines comparativos se usaron especímenes depositados en el Museo de Historia Natural de Londres, Inglaterra (NHMUK) y en el Museo Nacional de Historia Natural de Paris, Francia (MNHN).

\section{Resultados}

\section{Familia Marginellidae Fleming, 1828}

\section{Género Volvarina Hinds, 1844}

Volvarina morrocoyensis Caballer, Espinosa \& Ortea especie nueva (Fig. 1 A-B y Fig. 2 A-E, Tabla 1)

\section{Diagnosis}

Concha pardo amarillenta a blanca rosada, cruzada por 5 bandas espirales pardas, lisa, de tamaño pequeño a mediano, subcilíndrica, con la espira corta y el ápice saliente. Pliegues de la columela blancos, alineados y paralelos por pares, siendo el segundo el mayor de todos y oblicuo al primero en su parte terminal. Espira pardo rosada, con la región apical más clara. Cuerpo blanco opaco, con manchas rojas y blancas refringentes. Tentáculos con una mancha roja pegada al ojo. Manto con manchas negras que pueden llegar a formar 3 bandas.

\section{Material eXAminado}

Holotipo: El Ocho (localidad tipo: $10^{\circ} 52^{\prime} 1,44^{\prime \prime N}$; $\left.68^{\circ} 13^{\prime} 41,83^{\prime \prime O}\right)$, PNM, Venezuela (23/03/2010); ejemplar $(7,2 \times 3,65 \mathrm{~mm})$ recolectado vivo en una laguna costera rodeada de mangle rojo, en fondo arenoso-fangoso con Caulerpa spp. a $1 \mathrm{~m}$ de profundidad, preservado en seco y depositado en SOM-IVIC (IVICCMT012). Paratipo 1: 


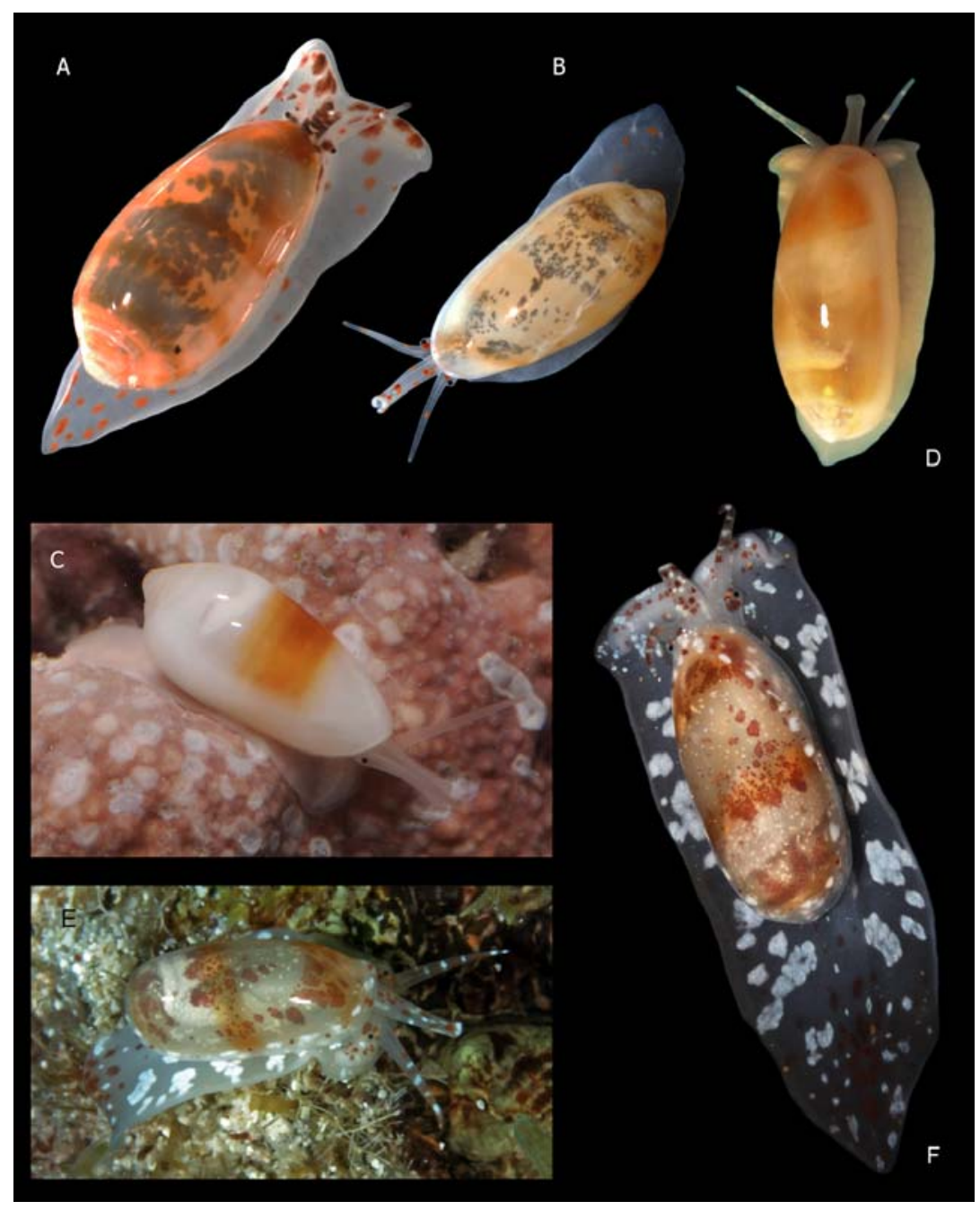

Figura 1. Ejemplares vivos de: A-B) Volvarina morrocoyensis, Caballer, Espinosa \& Ortea, especie nueva, A. holotipo, B. paratipo 3, C) Volvarina monchoi Caballer, Espinosa \& Ortea, especie nueva, holotipo. D) Volvarina avesensis Caballer, Espinosa \& Ortea, especie nueva, holotipo. E-F) Hyalina nelsyae especie nueva, E. vista lateral, F. vista dorsal / Living specimens of: A-B) Volvarina morrocoyensis, Caballer, Espinosa \& Ortea, new species, A. holotype, B. paratype 3, C) Volvarina monchoi Caballer, Espinosa \& Ortea, new species, holotype, D) Volvarina avesensis Caballer, Espinosa \& Ortea, new species, holotype. E-F) Hyalina nelsyae, new species, E. lateral view, F. dorsal view

\section{DESCRIPCIÓN}

Concha lisa y brillante, de tamaño pequeño a mediano, comparada con otras especies antillanas del género, de forma subcilíndrica, con la espira corta y el ápice saliente. Lado izquierdo de la concha (vista ventral) convexo, lado derecho menos convexo y ligeramente sinuoso hacia su parte media-baja anterior. Espira corta, ancha y saliente, formada por 2 vueltas de las cuales, la primera, grande, redondeada, saliente y provista de un núcleo relativamente pequeño y poco conspicuo respecto al resto del área apical, es de protoconcha. Protoconcha paucispiral, pequeña, lisa y blanco-amarillenta translúcida. Teleoconcha de 2 vueltas y media, la última ocupa entre el 91,4 y el $94,5 \%$ del largo total de la concha. Abertura casi tan larga como la última vuelta, estrecha en su porción posterior y algo más ancha en la anterior; labio externo 
Tabla 1. Medidas de los ejemplares estudiados de Volvarina e Hyalina / Measurements of the studied specimens of Volvarina and Hyalina

\begin{tabular}{lrccc}
\hline Especie & $\begin{array}{c}\text { Longitud } \\
(\mathrm{mm})\end{array}$ & $\begin{array}{c}\text { Diámetro } \\
(\mathrm{mm})\end{array}$ & $\begin{array}{c}\text { Espira } \\
(\mathrm{mm})\end{array}$ & $\begin{array}{c}\text { Última vuelta } \\
(\mathrm{mm})\end{array}$ \\
\hline Volvarina morrocoyensis Caballer, & 7,20 & 3,65 & 0,50 & 6,70 \\
Ortea y Espinosa especie nueva & 3,40 & 1,65 & 0,30 & 3,10 \\
& 6,95 & 3,40 & 0,54 & 6,41 \\
& 7,71 & 3,37 & 0,43 & 7,28 \\
& & & & \\
Volvarina monchoi Caballer, Ortea & 4,72 & 2,24 & 0,47 & 4,25 \\
y Espinosa especie nueva & & & & \\
& & & & \\
Volvarina avesensis Caballer, & 7,20 & 3,10 & 0,55 & 6,65 \\
Ortea y Espinosa especie nueva & 6,90 & 3,15 & 0,49 & 6,41 \\
& 6,80 & 3,00 & 0,48 & 6,32 \\
& 7,95 & 3,50 & 0,56 & 7,39 \\
& 6,65 & 3,00 & 0,47 & 6,18 \\
& & & & \\
Hyalina pallida (Linnaeus, 1758) & 15,30 & 7,00 & 0,80 & 14,50 \\
& & & & \\
Hyalina nelsyae especie nueva & 14,20 & 7,45 & 0,77 & 13,43 \\
& 7,95 & 5,35 & 0,43 & 7,52 \\
& 11,55 & 5,25 & 0,63 & 10,92 \\
& 7,30 & 3,15 & 0,40 & 6,90 \\
& 13,75 & 6,10 & 0,75 & 13,00 \\
& 6,20 & 5,05 & 0,44 & 5,76 \\
& 7,65 & 5,40 & 0,41 & 7,24 \\
& 12,90 & 5,00 & 0,70 & 12,20 \\
& 11,60 & 4,90 & 0,63 & 10,97 \\
& 14,10 & 5,50 & 0,76 & 13,34 \\
\hline & & & &
\end{tabular}

varicoso, relativamente estrecho y poco engrosado, insertado en la espira justo sobre la sutura de la vuelta precedente.

Columela con 4 pliegues, alineados y paralelos por pares entre sí, los 2 anteriores más desarrollados que los 2 posteriores, principalmente el segundo que es el mayor de todos y se acerca al primero en su parte terminal.

Concha con un color de fondo pardo amarillento a blanco rosado, cruzada en la última vuelta por 5 bandas espirales pardas, más o menos oscuras según los ejemplares, que esbozan pequeñas manchas pardas al final del labrum, el cual es blanco en casi su totalidad, al igual que los pliegues columelares. Espira pardo rosada, con la región apical más clara.

Cuerpo del animal de color blanco opaco, algo hialino por zonas, con manchas rojas, redondeadas o algo irregulares en el sifón, en los tentáculos y en la región anterior y posterior del pie, en cuyos laterales puede haber también pequeñas manchas blancas refringentes además de las rojas. Los tentáculos siempre presentan una mancha roja pegada al ojo y a veces pequeñas manchas blanco nieve. Borde anterior del pie partido en 2 lóbulos siendo el superior más corto que el inferior y los laterales redondeados. Manto en el interior de la concha con una tonalidad crema o anaranjada y manchas negras que pueden llegar a formar 3 bandas con pequeñas manchas intermedias entre ellas.

La rádula del paratipo 2 es una cinta con 48 placas de 130-150 $\mu \mathrm{m}$ de ancho, de las cuales las 8 primeras tienen color ambarino; en cada placa hay una cúspide central y 9 laterales, siendo el tamaño de la central el doble que el de la mayor de las cúspides laterales.

\section{Etimología}

Volvarina morrocoyensis, toponímico del lugar en el que se encuentra su localidad tipo, el Parque Nacional Morrocoy.

\section{ObSERVACIONES}

Por su tamaño pequeño y por su patrón de color, que se caracteriza por presentar 5 bandas espirales oscuras sobre la última vuelta, más o menos marcadas, Volvarina 
Figura 2. A-E) Volvarina morrocoyensis Caballer, Espinosa \& Ortea, especie nueva, A-C. holotipo, D-E. paratipo 2, A. vista ventral y dorsal, B. detalle de los pliegues, C. protoconcha, D. vista ventral y dorsal, E. placa radular. F-H) Volvarina monchoi Caballer, Espinosa \& Ortea, especie nueva, holotipo, F. vista ventral y dorsal, G. detalle de los pliegues, H. protoconcha. I-K) Volvarina avesensis Caballer, Espinosa \& Ortea, especie nueva, holotipo, I. vista ventral y dorsal, J. detalle de los pliegues, K. protoconcha / A-E) Volvarina morrocoyensis Caballer, Espinosa \& Ortea, new species, A-C. holotype, D-E. paratype 2, A. ventral and dorsal view, B. detail of the folds, C. protoconch, D. ventral and dorsal view, E. radular tooth. F-H) Volvarina monchoi Caballer, Espinosa \& Ortea, new species, holotype, F. ventral and dorsal view, G. detail of the folds, H. protoconch. I-K) Volvarina avesensis Caballer, Espinosa \& Ortea, new species, holotype, I. ventral and dorsal view, J. detail of the folds, $\mathrm{K}$. protoconch

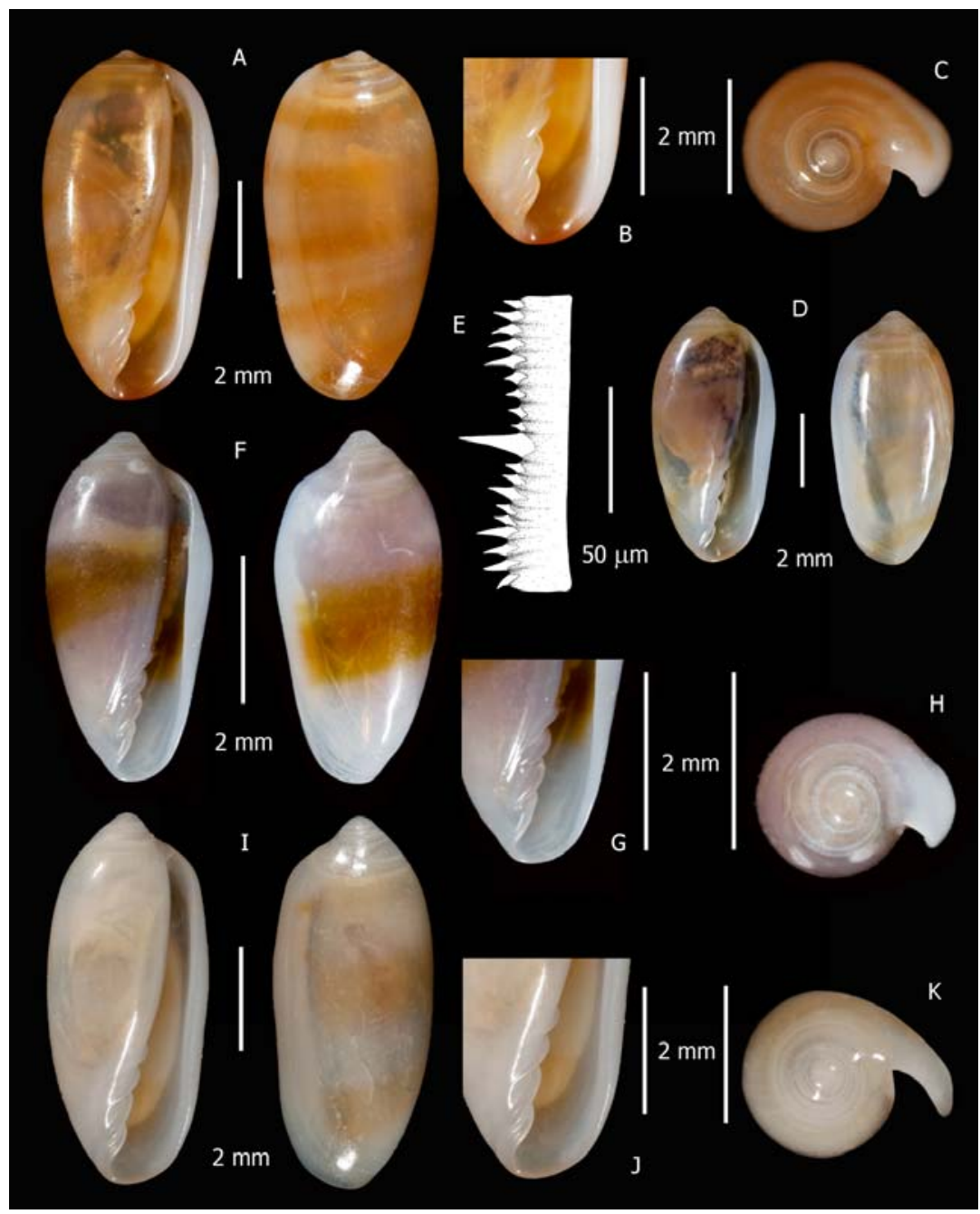

morrocoyensis, Caballer, Espinosa \& Ortea especie nueva, puede ser comparada con Volvarina isabelae (Borro, 1946), descrita de la zona occidental de la costa norte de Cuba, con Volvarina yolandae Espinosa \& Ortea, 2000, procedente de Manzanillo, en la costa caribeña de Costa Rica, Volvarina virginieae Espinosa \& Ortea, 2012 y con Volvarina caballeri Espinosa \& Ortea, 2012, ambas de la isla de Guadalupe. $V$. isabelae es de tamaño más pequeño (holotipo: 4,3 x 2,4 mm), con la espira corta y ampliamente redondeada (Borro 1946), lo que le confiere una forma muy diferente a la concha. V. yolandae, aunque es de tamaño similar (6,4 mm de largo), es comparativamente más alargada y estrecha, con sólo 2,5 mm de ancho; además tiene la espira más ancha y redondeada y presenta una marcada sinuosidad en casi todo el labio palatal. Adicionalmente, los pliegues columelares de ambas especies también son diferentes a los de esta nueva especie y la rádula de Volvarina yolandae presentó placas de $100 \mu \mathrm{m}$ de ancho y 21 cúspides (una central gruesa) en un animal de 5,4 mm, mientras que en Volvarina morrocoyensis Caballer, Espinosa \& Ortea especie nueva, las placas son más anchas (hasta $150 \mu \mathrm{m}$ ) y hay 19 cúspides; además, en V. yolandae la estructura de las cúspides laterales es simétrica en relación a la cúspide central y asimétrica en $V$. morrocoyensis. Volvarina virginieae Espinosa \& Ortea, 2012 se diferencia de $V$. morrocoyensis por presentar una concha con menos vueltas, menos simétrica en sus lados y menos redondeada, con el labio insertado en la espira por encima de la sutura de la vuelta precedente y manchado con la prolongación de las 5 bandas de la teleoconcha, con el callo postlabral extendido casi hasta el ápice, con los pliegues casi paralelos entre si y el animal blanco, con manchas blancas y solo algunos pequeños puntos rojos 
en el sifón (Espinosa \& Ortea 2012). Volvarina caballeri Espinosa \& Ortea, 2012 se diferencia de V. morrocoyensis por presentar una concha más pequeña, con menos vueltas, con la teleoconcha ocupando tan solo el $85,8 \%$ de la longitud total, con el labio insertado en la espira por encima de la sutura de la vuelta precedente y marcado en su parte dorsal con manchas pardas producto de las 2 bandas anteriores de la teleoconcha, con la columela con el primer pliegue más grande y el último reducido y el animal blanco, casi totalmente cubierto por manchas pardas oscuras y blancas refringentes (Espinosa \& Ortea 2012).

Por la forma general de la concha, Volvarina morrocoyensis, Caballer, Espinosa \& Ortea especie nueva, también puede ser comparada con Volvarina rubella (C.B. Adams, 1845) sensu Cossignani (2006), descrita en Jamaica, y con Volvarina adrianadiae Cossignani, 2006, de la Guajira, Colombia. Tal y como señalan Wakefield et al. (2002), V. rubella no ha vuelto a ser capturada en Jamaica desde su descripción original pero está bien representada en el sur del Caribe, además, no existe un tipo de esta especie, ni ilustración para los ejemplares del norte del Caribe. La determinación del resto de las citas de V. rubella se basan en el texto original: concha esbelta, alargada, con tres bandas de ornamentos rojos; 4 vueltas, espira cristalina, labro blanco, decorado con tres manchas rojizas, apertura estrecha en la parte superior, columela con 4 pliegues. Divergencia $100^{\circ}$. Longitud de la espira 0,6 mm. Longitud total 8,9 mm. Anchura 4,3 mm. (Adams 1845), y en material del sur del Caribe. De Jong \& Coomans (1988) afirman tener varios ejemplares de Aruba que encajan en los datos aportados por Adams (1845), que van de los 8,5 a los $12,5 \mathrm{~mm}$, algunos con 3 bandas y otros sin ellas, sin embargo no los ilustran. Cosignani (2006) publica una fotografía de V. rubella procedente del Caribe (sin localidad específica) de $12,5 \mathrm{~mm}$. Pese a lo dudoso que resulta que los ejemplares del sur del Caribe pertenezcan a la misma especie que los de Jamaica, se decidió asumir la foto de Cossignani (2006) como representación de $V$. rubella, hasta que sea nuevamente descrita con muestras de la localidad tipo. V. rubella se distingue de $V$. morrocoyensis por tener una concha mucho más grande (con igual número de vueltas), cilíndrica, alargada y gruesa, labio engrosado con 3 manchas, con coloración rojiza y 3 bandas en la teleoconcha en lugar de las 5 de $V$. morrocoyensis. $V$. adrianadiae tiene una forma similar a $V$. morrocoyensis, sin embargo, la concha es más alargada, blanca, sin bandas ni coloración alguna, con el labio más engrosado, deprimido en su zona media y con el borde posterior del labio levantado casi a la misma altura que el ápex, la espira mucho más corta en animales del mismo tamaño que el holotipo (Cossignani 2006) y el primer pliegue de la columela y la parte anterior de la concha mucho más engrosados.

Volvarina vokesi De Jong \& Coomans, 1988 citada en el PN Morrocoy por Wakefield, Boyer \& McCleery 2002, es una especie totalmente distinta de Volvarina morrocoyensis Caballer, Espinosa \& Ortea especie nueva, con una concha ahusada, blanca refringente con una banda media pardo amarillenta, con la espira alta y la inserción del labio bastante por debajo de la sutura de la vuelta precedente.

Volvarina monchoi Caballer, Espinosa \& Ortea especie nueva (Fig. 1 C y Fig. 2 F-H, Tabla 1)

\section{Diagnosis}

Concha blanca rosácea muy pálida, cruzada en su parte media por una ancha banda espiral de color pardo amarillento oscuro, lisa, subfusiforme, de tamaño pequeño a mediano. Espira algo estrecha y saliente. Protoconcha con un núcleo grande. Labio externo blanco opaco y poco engrosado. Pliegues de la columela desiguales, los 2 anteriores más desarrollados, particularmente el primero más grande. Cuerpo blanco hialino uniforme, sin manchas.

\section{Material EXAMinado}

Holotipo: Lado este de Playa el Yaque (localidad tipo: $\left.10^{\circ} 57^{\prime} 48^{\prime \prime} \mathrm{N} ; 65^{\circ} 13^{\prime} 30^{\prime \prime} \mathrm{O}\right)$, Isla La Tortuga, Venezuela (18/ 03/2010); ejemplar (4,72 x 2,24 mm) recolectado vivo en fondos con cantos sueltos y arena, batidos por el oleaje, entre 0 y $1 \mathrm{~m}$ de profundidad, preservado en seco y depositado en SOM-IVIC (IVICCMT015).

\section{DESCRIPCIÓN}

Concha de tamaño pequeño a mediano, comparada con otras especies antillanas del género, lisa y brillante, subfusiforme, con el lado izquierdo convexo y el derecho ligeramente sinuoso (en vista oral). Espira moderadamente extendida, algo estrecha y saliente, formada por algo más de 2 vueltas de las cuales, la primera, provista de un núcleo notable, es de protoconcha; la tercera y última vuelta ocupa aproximadamente el $90 \%$ del largo total de la concha (estimado del largo de la última vuelta $=4,25 \mathrm{~mm}$ ). Protoconcha paucispiral, grande, lisa, redondeada, blanca y de perfil elevado en su 
enrollamiento. Abertura alargada y estrecha, algo más ensanchada en su porción anterior, con el labio externo moderadamente ancho y poco engrosado, insertado en la espira justo por debajo la sutura de la vuelta precedente. Columela con 4 pliegues desiguales bien señalados, los 2 anteriores más desarrollados que los posteriores, sobre todo el primero que es el mayor, mientras que el cuarto pliegue posterior es el más débil y algo interno. Concha ligeramente translúcida, de color blanco rosáceo muy pálido, cruzada en la parte media de la última vuelta por una ancha banda espiral de color pardo amarillento oscuro. El labio externo es blanco opaco y los pliegues parecen reflejar el color de fondo de la concha, al igual que la espira, pero con la región apical más clara.

Cuerpo del animal de color blanco hialino uniforme, sin ningún tipo de mancha distintiva. Tentáculos y sifón del mismo color que el cuerpo, sin manchas. Borde anterior del pie partido en 2 lóbulos, el superior más corto que el inferior y con los laterales extendidos, triangulares y redondeados por delante. Cola tan larga como un tercio de la longitud de la concha.

\section{ETimología}

Nombrada en honor a 'Moncho', primer habitante de la isla de La Tortuga.

\section{Observaciones}

Considerando el tamaño y la forma general de la concha de Volvarina monchoi Caballer, Espinosa \& Ortea especie nueva, solamente guarda similitud con Volvarina vokesi De Jong \& Coomanss, 1988, descrita a partir de ejemplares de las islas de Aruba y Curaçao (los cuales podrían pertenecer a 2 especies distintas). V. vokesi tiene una concha con una banda espiral parda en la última vuelta similar a la de V. monchoi, pero también en la espira, es de mayor tamaño ( 5 a $6,3 \mathrm{~mm}$ de largo) y con los 4 pliegues columelares paralelos iguales en tamaño, muy diferentes de los de V. monchoi; además, la espira es mucho más elevada, sobre todo en el ejemplar de Curaçao de 6,3 mm ilustrado en la descripción original (De Jong \& Coomans 1988: Pl. 41, fig. 531B).

Otras 2 especies del género tienen su localidad tipo en la Isla La Tortuga; Volvarina ivic Caballer, Espinosa \& Ortea, 2009 y Volvarina latortuga Caballer, Espinosa \& Ortea, 2009, pero se distinguen con facilidad porque en ambos casos, sus conchas son mas grandes $(9,6$ y $6,5 \mathrm{~mm}$ de largo respectivamente) y ovoides, de color crema, con
3 bandas de color en la última vuelta y con los animales presentando manchas blancas o pardas, ya sea en el pie o en el dorso.

Volvarina avesensis Caballer, Espinosa \& Ortea especie nueva (Fig. 1 D y Fig. 2 I-K, Tabla 1)

\section{Diagnosis}

Concha blanca amarillenta pálida, cruzada por 3 bandas anchas anaranjadas, lisa, pequeña, subcilíndrica, alargada, con la espira corta y el ápice saliente. Protoconcha grande. Pliegues más o menos paralelos, los anteriores más desarrollados y sub-iguales, siendo el segundo es el más grande. Cuerpo blanco hialino, con un tinte anaranjado, sin manchas. Manto anaranjado con manchas castañas.

\section{Material eXAminado}

Holotipo: Isla de Aves (localidad tipo: $15^{\circ} 40^{\prime} 24,7^{\prime \prime} \mathrm{N}$; $\left.63^{\circ} 37^{\prime} 11^{\prime \prime O}\right)$, Venezuela (02/08/2010); ejemplar (7,2 x 3,1 $\mathrm{mm}$ ) recolectado vivo, fijado inicialmente en alcohol, posteriormente conservado en seco y depositado en las colecciones de FUDENA (CFRFSIA0004). Paratipo: Isla

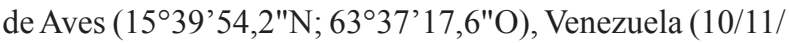
2009); concha (6,9 x 3,15 mm), depositada en las colecciones de FUDENA (CFRFSIA0005).

\section{Material Adicional}

Isla de Aves (15³9'54,2"N; 633’'17,6"O), Venezuela (10/ 11/2009); 9 conchas (de $6,8 \times 3 \mathrm{~mm}$ a $7,95 \times 3,5 \mathrm{~mm}$ ), depositadas en las colecciones de FUDENA. Isla de Aves $\left(15^{\circ} 40^{\prime} 24,7^{\prime \prime} \mathrm{N} ; 63^{\circ} 37^{\prime} 11^{\prime \prime O}\right)$, Venezuela (02/08/2010); ejemplar $(6,65 \times 3 \mathrm{~mm})$ recolectado vivo, fijado inicialmente en alcohol, posteriormente conservado en seco y depositado en las colecciones de FUDENA.

\section{DESCRIPCIÓN}

Concha lisa y brillante, de tamaño pequeño comparada con otras especies antillanas del género, de forma subcilíndrica alargada y estrecha, con la espira corta y el ápice saliente. Lado izquierdo de la concha (vista ventral) moderadamente convexo; el derecho casi recto. Protoconcha blanca translúcida, paucispiral, formada por al menos una vuelta grande, redondeada y saliente, con un núcleo relativamente pequeño. Teleoconcha de 2 vueltas y media, la última ocupa aproximadamente el $93 \%$ del largo total de la concha. Abertura casi tan larga como el largo total de la concha, estrecha en su porción posterior 
y algo más ancha en la anterior desde el comienzo de los pliegues columelares. Labrum relativamente estrecho, poco engrosado y formando un saliente en su zona media posterior, insertado en la espira justo sobre la sutura de la vuelta precedente, desde donde se extiende el callo post-labral casi hasta el ápice. Columela con 4 pliegues, más o menos paralelos entre sí, los 2 anteriores más desarrollados y sub-iguales (el segundo es el más grande). Concha con un color de fondo blanco amarillento pálido y algo translúcido, cruzada en la última vuelta por 3 bandas anchas espirales de color anaranjado pálido. El labio externo es blanco al igual que los pliegues. Espira blanca, translúcida.

Cuerpo del animal blanco hialino, con un tinte anaranjado uniforme, sin manchas destacadas. Sifón y tentáculos del mismo color que el cuerpo; los segundos con pequeñas manchas circulares internas de color blanco nieve o naranja. Borde anterior del pie partido en 2 lóbulos de color más intenso que el resto del cuerpo, con los laterales triangulares y redondeados por delante. En el interior de la concha el manto tiene una tonalidad crema anaranjada uniforme, con manchas castaño cerca de la sutura de la espira.

\section{Eтimología}

V. avesensis, toponímico de su localidad tipo, Isla de Aves.

\section{Observaciones}

Por el conjunto de los caracteres de la concha, Volvarina avesensis Caballer, Espinosa \& Ortea especie nueva, es comparable con Volvarina lineae Espinosa \& Ortea, 2012, con Volvarina grosi Espinosa \& Ortea, 2012 y con Volvarina alloginella Espinosa, Ortea \& Moro, 2011, del grupo de Volvarina avena (Valenciennes en Kiener, 1934), la cual engloba un complejo de especies no descritas en todo el Mar Caribe citadas bajo ese nombre (Espinosa et al. 2011), tal y como se aprecia en el trabajo de Cossignani (2006). La forma de la concha de V. avesensis, la separa de V. alloginella ya que esta última es más robusta y de tamaño mayor $(10,75 \mathrm{~mm}$, frente a los 7,95 mm de longitud máxima de $V$. avesensis). $V$. alloginella presenta una protoconcha parda amarillenta grande con un núcleo muy notable y una teleoconcha más pequeña que la de $V$. avesensis, siendo su concha más grande, con la abertura más corta y el labio externo más ancho y engrosado, el cual se inserta muy por debajo de la sutura de la vuelta precedente. Además, el desarrollo de los pliegues columelares es también diferente, siendo el primer pliegue de $V$. alloginella el más desarrollado. En relación al complejo avena, la cita de $V$. avena en isla de Aves (Coomans 1958) se debe sin duda a un error de identificación y debe ser repudiada, ya que, además de $V$. avesensis, no existe en esta localidad ninguna otra especie de Volvarina con caracteres semejantes.

Volvarina lineae Espinosa \& Ortea, 2012 se distingue de Volvarina avesensis Caballer, Espinosa \& Ortea especie nueva, por presentar una concha de mayor tamaño $(10,19 \times 4,11 \mathrm{~mm})$ con menos vueltas (3), con el eje axial ligeramente curvado hacia la derecha, la espira más larga, con la teleoconcha ocupando tan solo el $80,7 \%$ de la longitud total, el labio más engrosado, sin formar un saliente, insertado por debajo de la sutura de la vuelta precedente y el cuerpo del animal blanco, sin tinte anaranjado, pero con manchas naranja sobre el sifón y en el borde anterior del pie (Espinosa \& Ortea 2012).

Volvarina grosi Espinosa \& Ortea, 2012 es similar a V. lineae, y se distingue de Volvarina avesensis Caballer, Espinosa \& Ortea especie nueva, por presentar una concha de mayor tamaño $(9,6 \times 4,1 \mathrm{~mm})$ con menos vueltas (3), con la espira más larga, con la teleoconcha ocupando tan solo el $82,7 \%$ de la longitud total, el labio mucho más engrosado, pero sin formar un saliente, insertado notablemente por debajo de la sutura de la vuelta precedente, con los pliegues de la columela diferentes, siendo el mayor el primero, y el cuerpo del animal blanco, sin tinte anaranjado, pero con manchas naranja en la base de los ojos y en el borde anterior del pie (Espinosa \& Ortea 2012).

\section{GÉnero Hyalina Schumacher, 1817}

Hyalina pallida (Linnaeus, 1758) (Fig. 3 A-C, Tabla 1)

\section{Material eXaminado}

Neotipo: localidad indeterminada en las Indias occidentales, concha vacía $(15,3 \times 7 \mathrm{~mm})$, depositada en las colecciones del NHMUK (sin número).

\section{DESCRIPCIÓN}

Ver Coan \& Roth (1976).

\section{ObSERVACIONES}

Hyalina pallida (Linnaeus, 1758) ha sido citada en varias localidades en Venezuela (ver introducción) sin imágenes ni descripciones que permitan comparar las citas con el neotipo de la especie (Fig. 3 A-C), por lo que dichas citas 


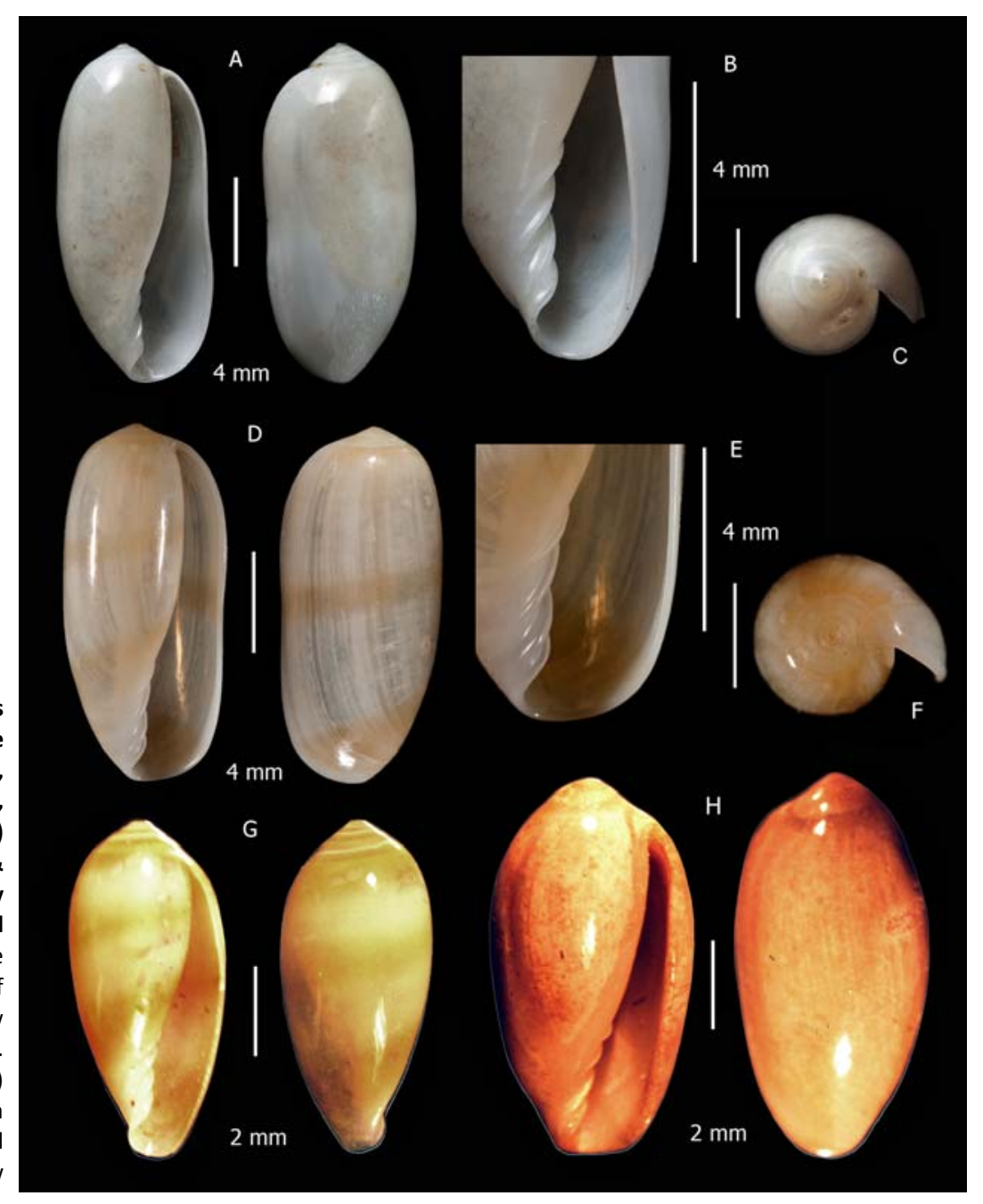

Figura 3. A-C) Hyalina pallida, nuevas ilustraciones del neotipo, A. vista ventral y dorsal, B. detalle de los pliegues, C. protoconcha. D-F) Hyalina nelsyae, especie nueva, holotipo, D. vista ventral y dorsal, E. detalle de los pliegues, F. protoconcha. G-H) Caribeginella flormarina (extraído de Espinosa \& Ortea 1998), G. holotipo de $7,5 \mathrm{~mm}$, vista ventral y dorsal. H. paratipo de $\mathbf{8 , 5} \mathbf{m m}$, vista ventral y dorsal ( A-C) Hyalina pallida, new illustrations of the neotype, A. ventral and dorsal view, B. detail of the folds, C. protoconch. D-F) Hyalina nelsyae, new species, holotype, D. ventral and dorsal view, E. detail of the folds, F. protoconch. G-H) Caribeginella flormarina (from Espinosa \& Ortea 1998), G. holotype $7.5 \mathrm{~mm}$, ventral and dorsal view. H. paratype $8,5 \mathrm{~mm}$, ventral and dorsal view

no pueden ser confirmadas. Coan \& Roth (1976) ilustran (en blanco y negro) y designan como neotipo (depositado en NHMUK) el ejemplar trabajado por Reeve (1866), quien señala que su hábitat son las Indias occidentales, sin concretar una localidad. Adicionalmente, Coan \& Roth (1976) describen la coloración de un animal vivo procedente de Water Island, en las Islas Vírgenes y depositado en las colecciones de la Academia de Ciencias de Filadelfia (ANSP 306669).

Recientemente, Fehse (2012) discute la clasificación supraespecífica y la validez de Caribeginella flormarina Espinosa \& Ortea, 1998, asignada originalmente en la Familia Triviidae por tener una rádula taeniglossa, y la considera un nomen dubium. Como justificación, dicho autor asume que, dado que $C$. flormarina tiene pliegues columelares y que estos no se encuentran en ningún Littorinimorpha, Espinosa \& Ortea (1998), debieron de mezclar o confundir la rádula publicada con la de un Triviidae o Eratoidae, es decir, que publicaron una rádula que no era de los animales descritos. Además, y aún sin basarse en dato alguno, afirma que Caribeginella parece sinónima de Hyalina por la morfología de la concha, siendo el holotipo (Fig. 3G) un sinónimo probable de $H$. pallida y el paratipo (Figura $3 \mathrm{H}$ ) una especie de Gibberula. La descripción original de C. flormarina incluye la ilustración de la concha y de las rádulas taeniglossas del paratipo $(8,5 \mathrm{~mm})$ y del holotipo $(7,5 \mathrm{~mm})$, este último con una fórmula radular: 14 x 2.1.R.1.2. Los ejemplares usados en dicho trabajo fueron colectados vivos (3 de los 4), todos en la misma localidad (Cayo Anclitas, Jardines de la Reina, Cuba) e inconfundibles por la morfología de los animales vivos; tipo 2 según la clasificación de Coovert \& Coovert (1995). La rádula de un tercer animal también se extrajo durante la descripción 
del género, por lo increíble del hallazgo, con los mismos resultados que en las otras dos (Ortea com. pers.). Espinosa y Ortea han descrito al menos 128 taxa de la Familia Marginellidae y 36 de la Familia Cystiscidae, es decir, algo más del $8 \%$ del total de las especies nombradas de ambas familias combinadas en toda la Historia (Bouchet \& Gofás 2012a; 2012b), resulta difícil de creer que hayan mezclado una Hyalina de 7,5 mm con rádula (las Hyalina no tienen rádula), con una Gibberula de $8,5 \mathrm{~mm}$ (sería de las más grandes registradas hasta la fecha) con la misma rádula que la anterior, para crear un género con ellas (Caribeginella) e incluirlas en la Familia Triviidae. La clasificación supra-genérica queda por resolver, pero Caribeginella es un género válido de animales marginelliformes con rádula taeniglossa y la sinonimia de C. flormarina con $H$. pallida debe ser desestimada. Probablemente la sinonimia de Fenhse (2012) se debe a las dificultades de este autor para entender la descripción original en español.

Hyalina nelsyae especie nueva (Fig. 1 E-F y Fig. 3 D-F, Tabla 1)

\section{Diagnosis}

Concha blanca rosada cruzada por 3 bandas pardoamarillentas, lisa, frágil, subcilíndrica alargada, con una ligera inflexión en su zona media. Labrum insertado sobre la sutura de la vuelta precedente. Pliegues marcados y blancos, divergentes los posteriores, casi paralelos los anteriores, mayores los 2 centrales. El segundo pliegue (el más grande) confluye con el primero. Cuerpo blanco hialino con manchas blancas, naranjas y rojizas, estas últimas forman un diamante en la cola. El manto en el interior de la concha forma 3 bandas rojizas.

\section{Material EXAMinado}

Holotipo: Isla de Aves (localidad tipo: $15^{\circ} 39^{\prime} 54,2^{\prime \prime N}$; $\left.63^{\circ} 37^{\prime} 17,6^{\prime \prime O}\right)$, Venezuela (10/11/2009); concha vacía (14,2 x 7,45 mm), depositada en las colecciones de FUDENA (CFRFSIA0006). Paratipo 1: Isla de Aves (15³9’54,2"N; $\left.63^{\circ} 37^{\prime} 17,6^{\prime \prime O}\right)$, Venezuela (10/11/2009); concha vacía (7,95 x 5,35 mm), depositada en SOM-IVIC (IVICCMT016).

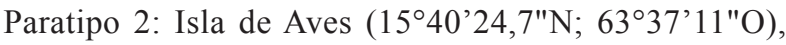
Venezuela (02/08/2010); concha vacía (11,55 x 5,25 mm), depositada en las colecciones de FUDENA (CFRFSIA0007).

\section{Material adicional}

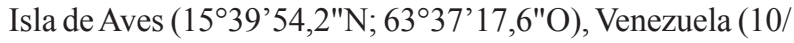
11/2009); 18 conchas (de 7,3 x 3,15 mm a 13,75 x 6,1 mm), depositadas en las colecciones de FUDENA. Isla de Aves (1540'24,7"N; 6337'11"O), Venezuela (02/08/2010); 2 conchas (de 6,2 x 5,05 mm a 7,65 x 5,4 mm), depositadas en las colecciones de FUDENA. Isla de Aves (15'39' $59,3^{\prime \prime} \mathrm{N}$; $\left.63^{\circ} 37^{\prime} 07,2^{\prime \prime O}\right)$, Venezuela (28/07/2012); 1 adulto (12,9 x 5 $\mathrm{mm}$ ) colectado vivo a $6 \mathrm{~m}$ de profundidad sobre macroalgas del género Dictyota Lamouroux, 1809 en fondo rocoso y depositado en las colecciones de FUDENA. Isla de Aves, plataforma rocosa noreste $\left(15^{\circ} 40^{\prime} 12,5^{\prime \prime} \mathrm{N} ; 63^{\circ} 37^{\prime} 05^{\prime \prime O}\right)$, Venezuela (28/07/2012); 5 conchas (de 11,6 x 4,9 mm a 14,1 x 5,5 mm), depositadas en las colecciones de FUDENA.

\section{DESCRIPCIÓN}

Concha lisa y brillante, algo translúcida y de aspecto frágil, de forma subcilíndrica alargada, con el lado izquierdo moderadamente convexo y el derecho casi recto, presentando sólo una ligera inflexión hacia su zona media. Espira corta y saliente, formada por algo más de 2 vueltas, la primera de las cuales, grande, redondeada y con un núcleo señalado, es de protoconcha; la tercera y última vuelta ocupa aproximadamente el $94,6 \%$ del largo total de la concha. Protoconcha lisa, paucispiral, grande y de perfil bajo. Abertura casi tan larga como la última vuelta, estrecha en su porción posterior y ancha en la anterior. Labrum estrecho, poco engrosado, ligeramente deprimido en su zona media e insertado en la espira justo sobre la sutura de la vuelta precedente. Columela con 4 pliegues marcados, los 2 centrales más desarrollados que los de los extremos, sobre todo el segundo anterior que es el mayor; los 2 pliegues posteriores son divergentes entre sí, mientras que los 2 anteriores son casi paralelos pero el final del segundo es confluente con el primero, reforzando ambos el extremo basal de la concha. Concha translúcida en animales medianos, blanco rosada hialina en los ejemplares de mayor tamaño, en ocasiones formando estrías que parecen marcar las líneas de crecimiento, que se alternan con zonas translúcidas. Concha cruzada en la última vuelta por 3 bandas espirales anchas de color pardo amarillento claro, una subsutural, una media y la otra anterior. Pliegues columelares blancos. Núcleo de la protoconcha en el holotipo sombreado de pardo amarillento claro.

Cuerpo del animal de color blanco hialino con manchas irregulares de color blanco refringente, mayores y más 
densas en la zona media y posterior de los laterales del pie y cuyo tamaño disminuye en el extremo de la cola donde se fragmentan y mezclan con algunos puntos naranjas y numerosas manchas irregulares pardo rojizas, que en conjunto forman un diamante.

Tentáculos y sifón del color del cuerpo, con manchas blanco refringente irregulares en el sifón y formando bandas en los tentáculos. Hay manchas pardo rojizas irregulares pero aparentes en el sifón, que están más o menos alineadas en el borde anterior del pie y formando bandas alternas en los rinóforos. Hay algunos puntos de color naranja, dispersos y poco evidentes, en la parte anterior del pie y flanqueando cada ojo en sus lados anterior y posterior, donde, en combinación con las manchas pardo rojizas, forman un diseño arriñonado.

Borde anterior del pie partido en dos labios, el superior más corto que el inferior; sus bordes laterales extendidos, son triangulares y angulosos. A través de la concha translucida con 3 bandas de color castaño claro se aprecia el manto del animal con un tinte verdoso pálido, que presenta pequeños puntos de color blanco refringente dispersos y uniformemente distribuidos, pero ninguno naranja, ni tampoco negro. Se observan grandes manchas de color pardo rojizo que se agrupan fundamentalmente siguiendo las 3 bandas de la concha. Una gran mancha de color blanco refringente es visible entre las bandas pardo rojizas media y posterior. El manto que cubre parte de la concha cuando el animal está relajado presenta bandas de color blanco refringente alternándose con puntos de color pardo rojizo.

\section{Etimología}

Nombrada en honor a Nelsy Rivero, malacóloga venezolana.

\section{ObSERVACIONES}

El género Hyalina es menos diverso que el género Volvarina y sus especies son más difíciles de separar debido a que sus conchas, en general, proporcionan menos información. Esta es posiblemente la causa de que, cualquier ejemplar colectado en el Caribe, presunta de pertenecer al género, fuera determinada como Hyalina pallida. Desafortunadamente, para la designación del neotipo de esta especie, Coan \& Roth (1976) usaron un espécimen del que no se conocía su localidad de colecta, sólo que era caribeño, una dificultad adicional a la hora de obtener nuevo material y establecer comparaciones. La primera especie de Hyalina descrita en el Caribe en el último siglo es Hyalina borroi Espinosa \& Ortea, 1998, de Cuba, aunque luego fue transferida a Volvarina por Espinosa \& Ortea (1999). A ésta le siguen Hyalina chicoi Espinosa \& Ortea, 1999, de Costa Rica, Hyalina cubensis Espinosa \& Ortea, 1999, Hyalina vallei Espinosa \& Ortea, 2002, Hyalina dearmasi Espinosa \& Ortea, 2003, Hyalina oscaritoi Espinosa, Moro \& Ortea, 2011, Hyalina surcaribe Espinosa, Moro \& Ortea, 2011, todas de Cuba, Hyalina redferni Espinosa \& Ortea, 2002, de Abaco, Bahamas y por último Hyalina moolenbeeki Espinosa \& Ortea, 2012, de Guadalupe.

Hyalina nelsyae, especie nueva, difiere del resto de los integrantes del género en el Caribe por la disposición y desarrollo de sus pliegues columelares, combinados con el patrón de coloración del cuerpo y el de la concha, que presenta 3 bandas. Hyalina pallida, con la que podría haber sido confundida en el pasado, es de mayor tamaño $(15,3 \mathrm{~mm}$ de largo $)$ y tiene menos vueltas, presentando una concha de color blanco uniforme, sin zonas hialinas, ni bandas, que tiene la espira más señalada, con una depresión más acentuada hacia la parte media del labio externo y unos pliegues columelares diferentes, con una notable depresión columelar entre los 3 pliegues más posteriores (Fig. 3 A-C). H. nelsyae especie nueva ha sido citada bajo el nombre $H$. pallida en Isla de Aves (Gibson-Smith 1972), su localidad tipo. Dicha cita debe ser corregida, siendo Hyalina nelsyae especie nueva, la única del género presente en el enclave.

Hyalina moolenbeeki es una especie próxima que tiene una coloración similar en la concha, presentando 3 bandas, sin embargo, se diferencia de Hyalina nelsyae especie nueva, por ser notablemente más ancha $(11,5 \times 6$ $\mathrm{mm}$ ), con la última vuelta ocupando el 90,3\% del largo total de la concha, por tener el labio redondeado, no deprimido en su zona media e insertado por debajo de la sutura de la vuelta precedente, por los pliegues de la columela, que ocupan una proporción menor del largo de la abertura, formando una depresión en esa zona, con 2 pliegues anteriores que no llegan a juntarse, por la coloración del animal, que es blanco y carece de manchas rojas, pero si tiene manchas blancas (en menor proporción), naranjas (en mucha mayor proporción) y negras (no existen en H. nelsyae especie nueva), estas últimas formando una banda bien definida en la cola (no un diamante). Además, el manto en el interior de la concha es de color crema y tiene manchas negras (no rojas, ni blancas) que no forman bandas como en $H$. nelsyae especie nueva. 


\section{Discusión}

Recientemente Caballer et al. (2009) recopilaron la lista de las especies de Volvarina citadas en Venezuela, estableciendo una base sobre la que esclarecer la verdadera identidad y la distribución de las especies del género en el país. La carencia de datos o ilustraciones asociada a algunas de esas citas y ciertas actualizaciones taxonómicas recientes hacen recomendable una breve revisión:

1. Volvarina ivic Caballer, Espinosa \& Ortea, 2009 y Volvarina latortuga Caballer, Espinosa \& Ortea, 2009, han sido descritas de forma detallada, con ilustraciones de las conchas y los animales vivos, quedando sus distribuciones conocidas limitadas al cayo La Herradura (isla La Tortuga) y al sur de la isla La Tortuga, respectivamente.

2. Volvarina avena (Kiener, 1834) ha sido citada en Isla de Aves (Coomans, 1958), archipiélago de los Roques, La Orchila (Gibson-Smith \& Gibson-Smith, 1972), Playa Grande (Vargas) (Talavera \& Princz 1985), Mochima (Sucre) (Ramos \& Robaina 1994), la isla de Margarita (Princz 1978, Macsotay \& Campos 2001), Cubagua, Araya (Macsotay \& Campos 2001), Chacopata (Sucre) (Narciso et al. 2002, Macsotay \& Campos 2001) y Las Rochas (Cossignani 2006). La cita en la Blanquilla atribuida a Talavera \& Princz (1985 fide Rosenberg 2009) por Caballer et al. (2009), se debe a un error de interpretación, ya que dichos autores especulan que la especie debería existir en la Blanquilla, pero sin afirmarlo y sin material en que basarse. Ninguna de estas citas es fiable ya que no van acompañadas de datos que permitan la identificación de los ejemplares, con excepción de la de Macsotay \& Campos (2001), que aportan una breve descripción, y la de Cossignani (2006), que aporta una foto. A lo largo de la historia reciente, cualquier Volvarina de concha alargada y estrecha, de color amarillento, con 3 bandas más oscuras en la última vuelta, con el labio comprimido en la zona media y 4 pliegues (los caracteres que da la descripción original), ha sido determinada como V. avena, es decir, el 50\% de las especies de Volvarina del Caribe. Algunas de ellas con cierto parecido a la ilustración original (Kiener 1834). Por desgracia, la localidad tipo de V. avena son las Indias Occidentales y no hay un tipo depositado en ninguna institución, así, el rango de distribución de la especie abarca desde Bermudas hasta Brasil (Rosenberg 2009), pero está basado en citas que son, en muchos casos, meros nombres en listas y que no se pueden contrastar. El ejemplar de Cossignani (2006) es similar a la ilustración original (Kiener 1834), no así los ejemplares trabajados por Macsotay \& Campos (2001), de los que dice que pueden llegar a tener hasta 6 bandas. Por tanto, puede considerarse que la especie está presente en Venezuela, aunque hay que confirmar su distribución.

3. Volvarina albolineata (d'Orbigny, 1842) ha sido citada en el archipiélago de los Roques por Talavera \& Princz (1985) y en Chacopata (Sucre) por Narciso et al. (2002), en ambos casos sin ilustraciones ni datos asociados. La localidad tipo de la especie es Cuba y dado que existen otras con las que se puede confundir por su coloración como: Volvarina ardovinii Cossignani, 1997, Volvarina bavecchii Cossignani, 2006 o la propia V. avena, consideramos que estas citas no son fiables.

4. Volvarina taeniata (Sowerby, 1846): citada en en la plataforma Margarita-Los Testigos, por Talavera \& Princz (1985), es una especie de Cabo Verde (Rolán 2005) cuya presencia en Venezuela debe ser rechazada.

5. Volvarina gracilis (CB Adams, 1851) ha sido citada en el Golfo de Santa Fé (Sucre), por Macsotay (1975), sin datos o ilustraciones que permitan su confirmación. La localidad tipo de esta especie es Jamaica; cita dudosa.

6. Volvarina mexicana (Jousseaume, 1875) es una especie cuya localidad tipo está es México, con una concha ovoide alargada con 4 vueltas, de color blanco porcelana con 4 bandas amarillas pálidas, con una apertura que abarca el total de la longitud de la concha (Jousseaume 1975). Caballer et al. (2009) afirman que esta especie ha sido citada en el Golfo de Venezuela por Lipe (1991) fide Rosenberg (2009), pero esto se debe a un error de interpretación, además, el ejemplar figurado por Lipe (1991) no se parece al ilustrado por Jousseaume (1875), ni al sintipo de la especie depositado en $\mathrm{MNHN}^{1}$. $V$. mexicana ha sido citada en el Golfo de Maracaibo (=Golfo de Venezuela), Venezuela, por Dautzenberg (1900), sin embargo este registro no va acompañado de ninguna ilustración ni de datos sobre los ejemplares. Yidi \& Sarmiento (2011) ilustran un ejemplar de la Bahía de Portete (una localidad a escasos $50 \mathrm{~km}$ del Golfo de Venezuela) atribuido a $V$. mexicana, que tampoco se parece al sintipo, ni encaja en la descripción original. Ya que dichos autores incluyen Venezuela en su distribución, es de suponer que se trate de la misma especie trabajada por Dautzenberg (1900) y por ello la cita V. mexicana en Venezuela ha de ser repudiada.

${ }^{1} \mathrm{MNHN}$ 1136:<http://coldb.mnhn.fr/CatalogNumber/MNHN/ IM/1136> 
7. Volvarina vokesi De Jong \& Coomans, 1988 ha sido citada en el Parque Nacional Morrocoy, Falcón, por Wakefield et al. (2002) como un nombre en una discusión, sin datos, ni ilustraciones, sin embargo, dada la proximidad de la localidad tipo (Aruba) y que dichos autores han tenido contacto con los tipos de la especie, consideramos que es una cita fiable.

8. Volvarina lactea (Kiener, 1841): El nombre Marginella lactea Kiener, 1841 (Volvarina), está preocupado por Marginella lactea Swainson, 1840 (una especie de Prunum Herrmannsen, 1852) y el primer nombre válido para esta especie es Volvarina abbreviata (Adams, 1850), que tiene su localidad tipo en Jamaica (Adams 1850, Rosenberg 2009). La cita de esta especie en la isla de Margarita por Macsotay \& Campos (2001: como $V$. lactea), se acompaña de una foto en blanco y negro bastante oscura y borrosa, que no permite observar bien los caracteres de la concha, pero también de una descripción idéntica a la dada por Adams (1850), por tanto, se ha de considerar válida esta cita.

9. Volvarina sp. 4 citada en la isla de Coche por Cossignani (2006) es probablemente la misma especie que la ilustrada por Macsotay \& Campos (2001) bajo el nombre $V$. lactea (=V. abbreviata), solo que estos últimos la representan inclinada para ver con facilidad los pliegues.

10. Hyalina (Volvarina) lustra Weisbord, 1962 es una especie descrita en base a un fósil procedente de la Quebrada Mare Abajo (Weisbord 1962), que fue incluida en la lista de sinónimos de $V$. lactea por Macsotay \& Campos (2001), quienes argumentaron que se parecía a los juveniles por ellos capturados en Margarita. Al examinar las ilustraciones que acompañan a la descripción original de esta especie se puede apreciar que se trata de un juvenil de $3,5 \mathrm{~mm}$, que podría pertenecer al género Hyalina y cuyo pliegue mayor de la columela es el cuarto y último, cuando en la descripción original de $V$. abbreviata se especifica claramente que dicho pliegue es pequeño (Adams 1950) en un ejemplar de 5,5 mm, por tanto, la sinonimia de $H$. lustra con $V$. lactea $(=V$. abbreviata) debe ser rechazada.

Las citas de Hyalina pallida (Linnaeus, 1758) en Venezuela también se basan en artículos en los que no se aporta información que permita identificar los ejemplares (Gibson-Smith 1972, Macsotay 1975, Talavera \& Princz 1985, Fernández \& Jiménez 2006, 2007). En Isla de Aves la presencia de $H$. pallida debe ser rechazada, ya que la especie que habita en dicha localidad es Hyalina nelsyae especie nueva. El resto de las citas son dudosas y dado que son escasas las referencias a esta especie en el sur del Caribe es probable que $H$. pallida no se encuentre en Venezuela.

En síntesis, y considerando los taxa descritos en este artículo, en Venezuela viven posiblemente 9 especies descritas del género Volvarina (V. avena, ¿V. gracilis?, V. lactea, V. vokesi, V. ivic, V. latortuga, Volvarina morrocoyensis, Caballer, Espinosa \& Ortea especie nueva, V. monchoi Caballer, Espinosa \& Ortea especie nueva y $V$. avesensis Caballer, Espinosa \& Ortea especie nueva) y 3 del género Hyalina (¿H. pallida?, H. lustra, H. nelsyae especie nueva).

\section{Agradecimientos}

A Moncho, que siempre nos brinda de forma desinteresada su hospitalidad y conocimientos cuando vamos a trabajar a la isla de la Tortuga y al resto de los pescadores, que siempre nos acogen y nos ayudan con los muestreos. A nuestro colega y amigo Leopoldo Moro por su ayuda con las fotografías de algunas de las conchas. A Gonzalo Medina por las fotos de los ejemplares vivos de V. avesensis. A Gabriela Carias por las fotos de los ejemplares vivos de $H$. nelsyae. A Kathie Way y a Harry Taylor (Unidad de Fotografía), del Museo de Historia Natural de Londres (NHMUK) por las ilustraciones y los datos del neotipo de Hyalina pallida. Al comandante de la Base Científico Militar Simón Bolívar en Isla de Aves, el TN Freddy Armada y a toda su tripulación. A los comandantes de los buques que nos transportaron a Isla de Aves y a sus tripulaciones: Patrullero de Vigilancia Oceánica (PC-23 Yekuana): CF Jesús Martin y Remolcador de Altura (RA 11): CF Antonio Mejía. Los muestreos necesarios para la realización de este trabajo fueron financiados parcialmente por el proyecto IVIC 915 (M.C.) - 'Biodiversidad marina en Venezuela y su relación con la dinámica costera. Los moluscos como grupo focal y fuente de nuevas moléculas'.

\section{LITERATURA CITADA}

Adams CB. 1845. Specierum novarum conchyliorum, in Jamaica repertorum, synopsis. Proceedings of the Boston Society of Natural History 2: 1-17.

Adams CB. 1850. Descriptions of supposed new species of marine shells, which inhabit Jamaica. Contributions to Conchology 4: 56-68.

Borro P. 1946. Una especie nueva de Marginella de Cuba. Revista de la Sociedad Malacológica 'Carlos de la Torre' 4: 41-42. 
Bouchet P \& S Gofás. 2012a. Marginellidae. World Register of Marine Species. <http://www.marinespecies.org/ aphia.php? $\mathrm{p}=$ taxdetails\&id $=23025>$

Bouchet P \& S Gofás. 2012b. Cystiscidae. World Register of Marine Species. <http://www.marinespecies.org/ aphia.php? $=$ taxdetails\&id $=23023>$

Caballer M, J Espinosa \& J Ortea. 2009. Dos nuevas especies del género Volvarina Hinds, 1844 (Mollusca: Neogastropoda: Marginellidae) de Venezuela. Revista de Biología Marina y Oceanografía 44(3): 533-538.

Coan EV \& B Roth. 1976. Status of the genus Hyalina Schumacher, 1817 (Mollusca: Gastropoda). Journal of Molluscan Studies 42: 217-222.

Coomans HE. 1958. A survey of the littoral gastropoda of the Netherlands Antilles and other Caribbean Islands. CARMABI, Curaçao. Collected Papers 6: 42-111.

Coovert GA \& HK Coovert. 1995. Revision of the supraspecific classification of the marginelliform gastropods. The Nautilus 109(2-3): 43-110.

Cossignani T. 2006. Marginellidae and Cystiscidae of the World, 408 pp. L'Informatore Piceno, Ancona.

Dautzenberg P. 1900. Croisières du yacht Chazalie dans 1'Atlantique: Mollusques. Meìmoires de la Socieìteì Zoologique de France 13: 145-265.

De Jong KM \& HE Coomans. 1988. Marine gastropods from Curaçao, Aruba and Bonaire. Studies on the Fauna of Curaçao and other Caribbean Islands 69: 1-261.

Espinosa J \& J Ortea. 1998. Nuevo género y nueva especie de Molusco Gasterópodo maginelliforme (Mollusca: Gastropoda) con rádula taenioglossa. Avicennia 8-9: 113116.

Espinosa J \& J Ortea. 1999. Nuevos datos anatómicos y posición sistemática de marginelas cubanas (Mollusca: Gastropoda: Marginellidae). Avicennia 10-11: 187-188.

Espinosa J \& J Ortea. 2001. Moluscos del mar Caribe de Costa Rica: desde Cahuita hasta Gandoca. Avicennia 4: 175 .

Espinosa J \& J Ortea. 2012. Nuevas especies de la Familia Marginellidae (Mollusca: Neogastropoda) de la isla Guadalupe (Karukera), Antillas Menores, mar Caribe. Revista de la Academia Canaria de Ciencias 24: 119-151.

Espinosa J, J Ortea, M Caballer \& L Moro. 2005. Nuevos datos sobre la familia Marginellidae (Mollusca: Neogratropoda) en Cuba, con la descripción de nuevas especies. Avicennia 18: 1-84.

Espinosa J, J Ortea \& L Moro. 2011. Nuevos datos sobre la familia Marginellidae (Mollusca: Neogratropoda) en Cuba, con la descripción de nuevas especies. Revista de la Academia Canaria de Ciencias 22(4): 161-188.
Fehse D. 2012. Caribeginella Espinosa \& Ortea 1998 eine Gattung der Trivioidea (Mollusca: Gastropoda: Littorinimorpha)? Conchylia 42(1-4): 49-50.

Fernández J \& M Jiménez. 2006. Estructura de la comunidad de moluscos y relaciones tróficas en el litoral rocoso del estado Sucre, Venezuela. Revista de Biología Tropical 54 (Supl. 3): 121-130.

Fernández J \& M Jiménez. 2007. Fauna malacológica del litoral rocoso de la costa sur del Golfo de Cariaco y costa norte del estado Sucre, Venezuela. Boletín del Instituto Oceanográfico de Venezuela 46(1): 3-11.

Gibson-Smith J \& W Gibson-Smith. 1972. A collection of molluscs from isla de Aves, Venezuela. In: Petzalli C (ed). Memorias de la VI Conferencia Geológica del Caribe, Isla de Margarita, Venezuela, pp. 470-478. CROMOTIP, Caracas.

Gofás S. 2011. Familias Cystiscidae y Marginellidae. En: Gofas S, D Moreno \& C Salas (eds). Moluscos marinos de Andalucía, pp. 318-323. Servicio de Publicaciones e Intercambio Científico, Universidad de Málaga, Málaga.

Jousseaume FP. 1875. Coquilles de la famille des marginelles. Revue et Magasin de Zoologie 3: 164-271.

Macsotay O. 1975. Geología submarina y fauna de metazoarios del Golfo de Santa Fe, Venezuela Nororiental. Boletín Geológico 12(23): 443-492.

Macsotay O \& R Campos. 2001. Moluscos representativos de la plataforma de Margarita, Venezuela, 280 pp. Rivolta, Valencia.

Narciso S, A Prieto \& V Acosta. 2002. Microgasterópodos asociados con el banco natural de la pepitona Arca zebra (Swaisson, 1833: Mollusca: Bivalvia) ubicado en la localidad de Chacopata, Estado Sucre, Venezuela. Ciencias Marinas 31(1A): 119-124.

Ponder WF. 1998. Family Marginellidae. In: Beesley PL, GJB Ross \& A Wells (eds). Mollusca: The Southern Synthesis. Part B. Fauna of Australia 5: 838-841. CSIRO Publishing, Melbourne.

Princz D. 1978. Moluscos gasterópodos y pelecípodos del Edo. Nueva Esparta, Venezuela. Memorias de la Sociedad de Ciencias Naturales de La Salle 50: 171-222.

Ramos H \& G Robaina. 1994. Contribución al conocimiento de los moluscos gasterópodos y pelecípodos de la bahía de Mochima, Estado Sucre, Venezuela. Memorias de la Sociedad de Ciencias Naturales de La Salle 54(141): 95106.

Reeve LA. 1866. Monograph of the genus Marginella. In: Reeve LA (ed). Conchologia Iconica: Or, Illustrations of Molluscous Animals 15: 48-160. Lovel Reeve \& Co, London. 
Rolán E. 2005. Malacological Fauna from the Cape Verde Archipelago, 455 pp. ConchBooks, Hackenheim.

Rosenberg G. 2009. Malacolog 4.1.1: A Database of Western Atlantic Marine Mollusca. <http://www.malacolog.org>

Talavera F \& D Princz. 1985. Marginella lasallei spec. nov. y revisión de la Familia Marginellidae en el mar venezolano. Bollettino Malacologico 20(9-12): 273-282.
Wakefield A, F Boyer \& T McCleery. 2002. Review of the genus Pachybathron Gaskoin, 1853 (Gastropoda: Cystiscidae). Novapex 3: 65-81.

Weisbord NE. 1962. Late Cenozoic gastropods from northern Venezuela. Bulletins of American Paleontology 42(193): 1672.

Yidi E \& V Sarmiento. 2011. Colombian Seashells from the Caribbean Sea, 400 pp. L'Informatore Piceno, Ancona.

Recibido el 27 de agosto de 2012 y aceptado el 1 de marzo de 2013

Editor Asociado: Mauricio Landaeta D. 